\title{
Common zeros of the solutions of two differential equations with transcendental coefficients
}

\author{
Asim Asiri ${ }^{1,2}$
}

\footnotetext{
Correspondence: amkasiri@kau.edu. sa

${ }^{1}$ Department of Mathematics, Faculty of Education, King Abdulaziz University, P.O. Box 15758, Jeddah 21454, Saudi Arabia Full list of author information is available at the end of the article
}

\begin{abstract}
Purpose: We consider a pair of homogeneous linear differential equations with transcendental entire coefficients of finite order, and the question of when solutions of these equations can have the same zeros or nearly the same zeros.

Method: We apply the Nevanlinna theory and properties of entire solutions of linear differential equations.

Conclusion: The results determine all pairs of such equations having solutions with the same zeros, or nearly the same zeros.

Keywords: Nevanlinna theory, differential equations
\end{abstract}

\section{Introduction}

This paper continues our study from [1] of the question of when two linear differential equations in the complex domain can have solutions with (nearly) the same zeros. Our starting point is the equation

$$
w^{\prime \prime}+P w=0,
$$

and [1] considered the case where $P$ is a polynomial. In this paper, $P$ will be a transcendental entire function, but we will retain the same notation since we will sometimes refer the reader to [1].

The following theorem was proved in 1955 by Wittich [2].

Theorem 1.1 If $f \neq 0$ is a non-trivial solution of $(1)$ and $P$ is a transcendental entire function, then we have:

(i) $f$ has infinite order and $T(r, P)=S(r, f)$.

(ii) If $a$ is a non-zero complex number, then $f$ takes the value a infinitely often, and in fact

$$
N\left(r, \frac{1}{f-a}\right)=T(r, f)+S(r, f) .
$$

In this paper, we use standard notation of Nevanlinna theory from [3]. The reader is referred to the books of Hille [4] and Laine [5], the keynote paper [6], and to [7-14] for comprehensive results on the zeros of solutions of linear differential equations with entire coefficients.

(c) 2011 Asiri; licensee Springer. This is an Open Access article distributed under the terms of the Creative Commons Attribution License (http://creativecommons.org/licenses/by/2.0), which permits unrestricted use, distribution, and reproduction in any medium, provided the original work is properly cited. 
The following lemma from [1] is needed in order to state our main results: the proof is by induction as in [1].

Lemma 1.1 Suppose $w^{\prime \prime}=-P w$ where $P$ is an entire function with order of growth $\rho$ $(P)<\infty$. Then for $j \geq 0$, there exist entire functions $Q_{j}$ and $R_{j}$ of finite order such that

$$
w^{(j)}=Q_{j} w+R_{j} w^{\prime}
$$

In particular,

$$
\begin{aligned}
& Q_{0}=1, \quad Q_{1}=0, \quad Q_{2}=-P, \quad Q_{3}=-P^{\prime}, \quad Q_{4}=P^{2}-P^{\prime \prime}, \\
& R_{0}=0, \quad R_{1}=1, \quad R_{2}=0, \quad R_{3}=-P, \quad R_{4}=-2 P^{\prime} .
\end{aligned}
$$

Now, our first main result can be stated.

Theorem 1.2 Let $P$ be a transcendental entire function with $\rho(P)<\infty$. Let $w \neq 0$ be a solution of (1). Assume that the zeros of $w$ have infinite exponent of convergence, i.e.

$$
\lambda(w)=\limsup _{r \rightarrow \infty} \frac{\log ^{+} N\left(r, \frac{1}{w}\right)}{\log r}=\infty .
$$

Let $v \neq 0$ be entire solution of the differential equation

$$
v^{(k)}+\sum_{1 \leq j \leq k-2} B_{j} v^{(j)}+A v=0, \quad k \geq 2
$$

such that $A$ and $B_{j}$ are entire functions and $\rho(A)<\infty$ and $\rho\left(B_{j}\right)<\infty$. Assume that $N$ (r) has finite order, where $N(r)$ counts both zeros and poles of $\frac{v}{w}$. Let

$$
v=L w .
$$

Then, one of the following two possibilities holds.

(a) $L$ is constant, and

$$
A=-Q_{k}-\sum_{j=1}^{k-2} B_{j} Q_{j}
$$

where $Q_{k}$ and $Q_{j}$ are deffined by Lemma 1.1.

(b) $L$ is not constant, but $L$ satisfies

$$
\sum_{m=0}^{k}\left[\left(\begin{array}{l}
k \\
m
\end{array}\right) L^{(m)} R_{k-m}+\sum_{j=1}^{k-2}\left(\begin{array}{l}
j \\
m
\end{array}\right) B_{j} L^{(m)} R_{j-m}\right]=0,
$$

and $A$ satisfies

$$
A=-\left(\sum_{m=0}^{k}\left[\left(\begin{array}{l}
k \\
m
\end{array}\right) \frac{L^{(m)}}{L} Q_{k-m}+\sum_{j=1}^{k-2}\left(\begin{array}{l}
j \\
m
\end{array}\right) B_{j} \frac{L^{(m)}}{L} Q_{j-m}\right]\right),
$$

where $R_{k-m}, R_{j-m}, Q_{k-m}$, and $Q_{j-m}$ are also defined by Lemma 1.1.

As in [1], we note that there is no loss of generality in assuming that there is no term in $w^{\prime}$ in (1) and that there is no term $B_{k-1}$ in (5), since we are considering zeros of solutions. 
We also note that the hypothesis (4) is not redundant: to see this, let $w=e^{B}$ and $v=$ $e^{C}$ where $B$ and $C$ are any entire functions of finite order. Then, $w$ and $v$ solve

$$
w^{\prime \prime}+P w=0, \quad v^{(k)}+Q v=0,
$$

where $P=-\left(B^{\prime \prime}+B^{\prime 2}\right)$ and $Q=-v^{(k)} / v=-\left(C^{\prime}\right)^{k}+\ldots$ are entire of finite order, but since $B$ and $C$ are arbitrary, there is no relationship between $P$ and $Q$.

The following results for the cases $k=2, k=3$ and $k=4$ will be deduced from Theorem 1.2.

Theorem 1.3 Let $P$ be a transcendental entire function and $\rho(P)<\infty$. Let $w \equiv 0$ be a solution of (1). Assume that the zeros of $w$ have infinite exponent of convergence, i.e. (4) holds. Let $v \neq 0$ be entire solution of the differential equation

$$
v^{\prime \prime}+A v=0,
$$

such that $A$ is an entire function and $\rho(A)<\infty$. Assume that $N(r)$ has finite order, where $N(r)$ counts zeros and poles of $\frac{v}{w}$. Then, $L=\frac{v}{w}$ is a constant and $A=P$.

Example 1.1 This example shows that $\rho(A)<\infty$ is vital in Theorem 1.3. To show this, let $v=w e^{g}$ where $g$ is an entire function. Then, we get

$$
\begin{aligned}
-A=\frac{v^{\prime \prime}}{v} & =\frac{w^{\prime \prime}}{w}+2 g^{\prime} \frac{w^{\prime}}{w}+g^{\prime \prime}+g^{\prime 2} \\
& =-P+g^{\prime \prime}+g^{\prime 2}+2 g^{\prime} \frac{w^{\prime}}{w} .
\end{aligned}
$$

Now, by putting $g^{\prime}=w$, we obtain

$$
-A=-P+w^{\prime}+w^{2}+2 w^{\prime} .
$$

Thus, $A$ is entire, $\frac{v}{w}$ is non constant and $v$ has the same zeros as $w$.

Theorem 1.4 Let $P$ be a transcendental entire function and $\rho(P)<\infty$. Let $w \equiv 0$ be a solution of (1). Assume that the zeros of $w$ have infinite exponent of convergence, i.e. (4) holds. Let $v \neq 0$ be entire solution of the differential equation

$$
v^{\prime \prime \prime}+B v^{\prime}+A v=0,
$$

such that $A$ and $B$ are entire functions with $\rho(A)<\infty$ and $\rho(B)<\infty$. Assume that $N$ (r) has finite order, where $N(r)$ counts zeros and poles of $\frac{v}{w}$. Then, $v=L w$ and one of the following holds.

(a) $L$ is constant and $A=P^{\prime}, B=P$.

(b) $L$ is non constant and $L^{\prime \prime}=\frac{1}{3} P L-\frac{1}{3} B L$ and

$$
A=\frac{8}{3} P \frac{L^{\prime}}{L}+\frac{2}{3} P^{\prime}+\frac{1}{3} B^{\prime}-\frac{2}{3} B \frac{L^{\prime}}{L} .
$$

Remark 1.1 If $B \equiv 0$ in Theorem 1.4 then case (a) cannot hold since P is transcendental.

Example 1.2 In this example, we show that case (b) can occur in Theorem 1.4. To show this, we can use Example 2.3 from [1] with $Q$ a transcendental entire function of finite order. 
Let $L=e^{Q}$ and set

$$
\frac{P}{3}=\frac{L^{\prime \prime}}{L}=Q^{\prime 2}+Q^{\prime \prime}
$$

If $w$ solves (1), then $v=L w$ satisffies

$$
v^{\prime \prime \prime}=L\left(-P^{\prime} w-P w^{\prime}\right)+3 L^{\prime}(-P w)+3 \frac{P}{3} L w^{\prime}+\left(\frac{P}{3} L^{\prime}+\frac{P^{\prime}}{3} L\right) w
$$

and so $v$ solves $v^{\prime \prime \prime}+A v=0$ with

$$
A=\frac{2}{3} P^{\prime}+\frac{8}{3} P Q^{\prime}
$$

It should be noted that $P$ is transcendental since $Q$ is transcendental and in fact writing

$$
Q^{\prime}=\frac{P}{3 Q^{\prime}}-\frac{Q^{\prime \prime}}{Q^{\prime}}
$$

shows that

$$
m\left(r, Q^{\prime}\right) \leq m(r, P)+S\left(r, Q^{\prime}\right),
$$

therefore,

$$
\rho(P)=\rho\left(Q^{\prime}\right)=\rho(Q) .
$$

Also, $P$ and $A$ have finite order.

Theorem 1.5 Let $P$ be a transcendental entire function and $\rho(P)<\infty$. Let $w \neq 0$ be a solution of (1). Assume that the zeros of $w$ have infinite exponent of convergence, i.e. (4) holds. Let $v \neq 0$ be entire solution of the differential equation

$$
v^{(4)}+A v=0,
$$

such that $A$ is an entire function with $\rho(A)<\infty$. Assume that $N(r)$ has finite order, where $N(r)$ counts zeros and poles of $\frac{v}{w}$. Then, $v=L w$ where $L$ is non-constatnt,

$$
L^{\prime \prime \prime}=L^{\prime} P+\frac{1}{2} L P^{\prime}, \quad A=5 P \frac{L^{\prime \prime}}{L}+\frac{5}{2} P^{\prime} \frac{L^{\prime}}{L}+\frac{1}{2} P^{\prime \prime}-P^{2}
$$

and $L=y_{1} y_{2}$ where $y_{1}, y_{2}$ are solutions of

$$
y^{\prime \prime}-\frac{P}{4} y=0
$$

In particular, if $v$ and $w$ have the same zeros with the same multiplicities, then $L$ is entire with no zeros and so are $y_{1}$ and $y_{2}$. In addition, when $v$ and $w$ have the same zeros:

(i) if $y_{1}, y_{2}$ are linearly dependent then $L=e^{2 C}$ with $C$ an entire function, $P=4\left(C^{\prime \prime}+\right.$ $C^{2}$ ) and $A$ is a differential polynomial in $C$;

(ii) if $y_{1}, y_{2}$ are linearly independent then $L=e^{C}$ with $C$ an entire function, $P=2 C^{\prime \prime}+$ $C^{2}+k^{2} e^{-2 C}$ where $k$ is a constant and $A$ is a differential polynomial in $e^{-C}$ and $C$. 
Example 1.3 To show that (13) can occur, let $L=Y^{2}=e^{Q}$ where $Q$ is a transcendental entire function of finite order and set

$$
Q^{\prime}=S=2 \gamma=2 \frac{Y^{\prime}}{Y}, \quad P=S^{2}+2 S^{\prime}=4\left(y^{2}+\gamma^{\prime}\right)
$$

so that $P$ is an entire function of finite order, and the same argument as in Example 1.2 shows that $P$ is transcendental. Then, as in Example 2.6 of [1],

$$
\begin{aligned}
L^{\prime} & =2 Y Y^{\prime}, \\
L^{\prime \prime} & =2 Y^{\prime 2}+2 Y Y^{\prime \prime}=2 Y^{\prime 2}+\frac{P}{2} L, \\
L^{\prime \prime \prime} & =P L^{\prime}+\frac{P^{\prime}}{2} L,
\end{aligned}
$$

If $w$ solves (1) then $v=L w$ satisfies

$$
\begin{aligned}
v^{(4)} & =L\left(\left(P^{2}-P^{\prime \prime}\right) w-2 P^{\prime} w^{\prime}\right)+4 L^{\prime}\left(-P w^{\prime}-P^{\prime} w\right)+6 L^{\prime \prime}(-P w) \\
& +4\left(P L^{\prime}+\frac{P^{\prime}}{2} L\right) w^{\prime}+\left(P L^{\prime \prime}+\frac{3}{2} P^{\prime} L^{\prime}+\frac{P^{\prime \prime}}{2} L\right) w
\end{aligned}
$$

and so $v$ solves (12) with

$$
A=5 P\left(Q^{\prime 2}+Q^{\prime \prime}\right)+\frac{5}{2} P^{\prime} Q^{\prime}+\frac{1}{2} P^{\prime \prime}-P^{2},
$$

which is also entire of finite order.

\section{Proof of Theorem $\mathbf{1 . 2}$}

In this proof, we use $M_{1}, M_{2}, \ldots$ to denote positive constants.

Claim 1: We claim that $w$ has simple zeros and

$$
N\left(r, \frac{1}{w}\right)=N\left(r, \frac{w^{\prime}}{w}\right)
$$

This holds by the existence-uniqueness theorem [4].

Lemma 2.1 There exists $N>0$ such that $m\left(r, \frac{w^{\prime}}{w}\right)<r^{N}$ as $r \rightarrow \infty$.

Proof: We can get this as follows. Use $N_{1}, N_{2}, \ldots$ to denote positive constants. Since $A$ has finite order,

$$
M(r, A) \leq \exp \left(r^{N_{1}}\right) \quad \text { as } r \rightarrow \infty .
$$

So for $r$ outside a set $E_{0}$ of finite logarithmic measure, we get from Wiman-Valiron theory [5]

$$
\left(\frac{v(r, w)}{r}\right)^{2} \leq(1+o(1)) M(r, A),
$$

where $v(r, w)$ denotes the central index, and so

$$
v(r, w) \leq \exp \left(r^{N_{2}}\right) \quad\left(r \rightarrow \infty, r \notin E_{0}\right)
$$


Hence, by [5, Lemma 1.1.2] and the fact that $v(r, w)$ is non-decreasing,

$$
v(r, w) \leq \exp \left(r^{N_{3}}\right) \quad \text { as } r \rightarrow \infty, \text { for all } r
$$

So the maximum term $\mu(r, w)$ satisfies

$$
\log \mu(r, w) \leq \exp \left(r^{N_{4}}\right)
$$

and so

$$
T(r, w) \leq \log M(r, w) \leq \exp \left(r^{N_{5}}\right)
$$

Now, we can use Lemma 2.3 in [[3], p. 36] with $R=2 r$ to get

$$
\begin{aligned}
m\left(r, \frac{w^{\prime}}{w}\right) & \leq O\left(\log ^{+} T(R, w)+\log r\right) \\
& \leq r^{N_{6}}
\end{aligned}
$$

This completes the proof of this lemma.

From (1) we have (2). From (2), (6) and by using Leibniz' rule, we get, for $1 \leq j \leq k$,

$$
\begin{aligned}
v^{(j)} & =\sum_{m=0}^{j}\left(\begin{array}{l}
j \\
m
\end{array}\right) L^{(m)}\left(Q_{j-m} w+R_{j-m} w^{\prime}\right) \\
& =\left(\sum_{m=0}^{j}\left(\begin{array}{l}
j \\
m
\end{array}\right) L^{(m)} Q_{j-m}\right) w+\left(\sum_{m=0}^{j}\left(\begin{array}{l}
j \\
m
\end{array}\right) L^{(m)} R_{j-m}\right) w^{\prime}
\end{aligned}
$$

From (5), (14) and the fact that $\left(\begin{array}{l}j \\ m\end{array}\right)=0$ when $j<m \leq k$, we find that

$$
\begin{aligned}
-A L w=-A v & =v^{(k)}+\sum_{1 \leq j \leq k-2} B_{j} v^{(j)} \\
& =\left(\sum_{m=0}^{k}\left(\begin{array}{l}
k \\
m
\end{array}\right) L^{(m)} Q_{k-m}\right) w+\left(\sum_{m=0}^{k}\left(\begin{array}{l}
k \\
m
\end{array}\right) L^{(m)} R_{k-m}\right) w^{\prime} \\
& +\sum_{j=1}^{k-2} B_{j}\left[\left(\sum_{m=0}^{j}\left(\begin{array}{l}
j \\
m
\end{array}\right) L^{(m)} Q_{j-m}\right) w+\left(\sum_{m=0}^{j}\left(\begin{array}{l}
j \\
m
\end{array}\right) L^{(m)} R_{j-m}\right) w^{\prime}\right] \\
& =\left[\sum_{m=0}^{k}\left(\begin{array}{l}
k \\
m
\end{array}\right) L^{(m)} Q_{k-m}+\sum_{j=1}^{k-2} B_{j}\left(\sum_{m=0}^{j}\left(\begin{array}{l}
j \\
m
\end{array}\right) L^{(m)} Q_{j-m}\right)\right] w \\
& +\left[\sum_{m=0}^{k}\left(\begin{array}{l}
k \\
m
\end{array}\right) L^{(m)} R_{k-m}+\sum_{j=1}^{k-2} B_{j}\left(\sum_{m=0}^{j}\left(\begin{array}{l}
j \\
m
\end{array}\right) L^{(m)} R_{j-m}\right)\right] w^{\prime} \\
& =\left(\sum_{m=0}^{k}\left[\left(\begin{array}{l}
k \\
m
\end{array}\right) L^{(m)} Q_{k-m}+\sum_{j=1}^{k-2}\left(\begin{array}{l}
j \\
m
\end{array}\right) B_{j} L^{(m)} Q_{j-m}\right]\right) w \\
& +\left(\sum_{m=0}^{k}\left[\left(\begin{array}{l}
k \\
m
\end{array}\right) L^{(m)} R_{k-m}+\sum_{j=1}^{k-2}\left(\begin{array}{l}
j \\
m
\end{array}\right) B_{j} L^{(m)} R_{j-m}\right]\right) w^{\prime} .
\end{aligned}
$$


Then, we have

$$
\begin{aligned}
0 & =\sum_{m=0}^{k}\left[\left(\begin{array}{l}
k \\
m
\end{array}\right) L^{(m)} Q_{k-m}+\sum_{j=1}^{k-2}\left(\begin{array}{l}
j \\
m
\end{array}\right) B_{j} L^{(m)} Q_{j-m}\right]+A L \\
& +\left(\sum_{m=0}^{k}\left[\left(\begin{array}{l}
k \\
m
\end{array}\right) L^{(m)} R_{k-m}+\sum_{j=1}^{k-2}\left(\begin{array}{l}
j \\
m
\end{array}\right) B_{j} L^{(m)} R_{j-m}\right]\right) \frac{w^{\prime}}{w} .
\end{aligned}
$$

Now, there are three cases which should be considered.

Case (I): If $L$ is a constant, then $w$ solves (5) and, by using Lemma 1.1, we obtain the following equations

$$
\left\{\begin{array}{l}
w^{(k)}+\sum_{1 \leq j \leq k-2} B_{j} w^{(j)}+A w=0 \\
-w^{(k)}+Q_{k} w+R_{k} w^{\prime}=0 .
\end{array}\right.
$$

By adding these two equations and using (2) again, we get

$$
0=\sum_{1 \leq j \leq k-2} B_{j}\left(Q_{j} w+R_{j} w^{\prime}\right)+A w+Q_{k} w+R_{k} w^{\prime} .
$$

Therefore, we can write

$$
\left(R_{k}+\sum_{1 \leq j \leq k-2} B_{j} R_{j}\right) \frac{w^{\prime}}{w}+A+Q_{k}+\sum_{1 \leq j \leq k-2} B_{j} Q_{j}=0 .
$$

Now, if $R_{k}+\sum_{1 \leq j \leq k-2} B_{j} R_{j} \equiv 0$, then $A+Q_{k}+\sum_{1 \leq j \leq k-2} B_{j} Q_{j} \equiv 0$ and so we have (7) and conclusion (a).

Suppose next that $R_{k}+\sum_{1 \leq j \leq k-2} B_{j} R_{j} \not \equiv 0$; then

$$
w=0 \Rightarrow \frac{w^{\prime}}{w}=\infty \Rightarrow R_{k}+\sum_{1 \leq j \leq k-2} B_{j} R_{j}=0 .
$$

Recall that all zeros of $w$ are simple. We deduce that

$$
\begin{aligned}
N\left(r, \frac{1}{w}\right) & \leq N\left(r, \frac{1}{R_{k}+\sum_{1 \leq j \leq k-2} B_{j} R_{j}}\right) \\
& =O\left(r^{M_{1}}\right) .
\end{aligned}
$$

But this contradicts (4).

Case (II): If $L$ is not constant and (8) holds, then from (15), we get (9) and conclusion (b) of the theorem.

It remains only to show that the following case cannot occur.

Case (III): Suppose that $L$ is not constant and (8) does not hold and let $S=L^{\prime} / L$.

We first compare $N(r, S)$ with $N(r)$. Recall that all zeros of $w$ are simple. On the other hand, $v$ solves a differential equation of order $k$. So, zeros of $v$ have multiplicities less than or equal to $k-1$.

So, $L=\frac{v}{w}$ has zeros with multiplicities at most $k-1$ and has simple poles. Then, we get 


$$
N(r, S) \leq N\left(r, \frac{1}{L}\right)+N(r, L)=N(r)
$$

Claim 2: We claim that

$$
T(r, S) \leq O\left(r^{M_{2}}\right)
$$

for $r$ outside a set $E$ of finite linear measure.

To prove this, we use the fact that $Q_{0}=1$ and $R_{0}=0$ in Lemma 1.1 to write (15) in the form

$$
\begin{aligned}
0 & =\frac{L^{(k)}}{L}+A \\
& +\sum_{m=0}^{k-1} \frac{L^{(m)}}{L}\left[\left(\begin{array}{l}
k \\
m
\end{array}\right)\left(Q_{k-m}+R_{k-m} \frac{w^{\prime}}{w}\right)+\sum_{j=1}^{k-2}\left(\begin{array}{l}
j \\
m
\end{array}\right) B_{j}\left(Q_{j-m}+R_{j-m} \frac{w^{\prime}}{w}\right)\right] .
\end{aligned}
$$

We can write, for $1 \leq m \leq k$,

$$
\frac{L^{(m)}}{L}=S^{m}+U_{m-1}(S)
$$

where $U_{m-1}(S)$ is a polynomial in $S, S^{\prime}, S^{\prime \prime}, \ldots, S^{(k)}$ with constant coefficients and total degree at most $m$ - 1. This follows immediately from Lemma 3.5 in [3] and can be easily proved by induction.

This gives us an integer $q>0$ such that (17) may be written as

$$
S^{k}=\sum_{j=0}^{q}\left(a_{j}+b_{j} \frac{w^{\prime}}{w}\right) S^{i_{0, j}}\left(S^{\prime}\right)^{i_{1, j}}\left(S^{\prime \prime}\right)^{i_{2, j}} \cdots\left(S^{(k)}\right)^{i_{k, j}}
$$

where $i_{\mu, j} \geq 0$ are integers and

$$
\sum_{\mu=0}^{k} i_{\mu, j} \leq k-1
$$

for each $j$. Lemma 2.1 gives $m\left(r, \frac{w^{\prime}}{w}\right)<r^{M_{3}}$. Also, $a_{j}$ and $b_{j}$ are polynomials in $A, B_{\mu}$, $Q_{\mu}$ and $R_{\mu}$, and so satisfy

$$
m\left(r, a_{j}\right)+m\left(r, b_{j}\right)=O\left(r^{M_{4}}\right) \quad \text { as } r \rightarrow \infty
$$

By Clunie's lemma [[5], p. 39], we should have

$$
m(r, S) \leq O\left(r^{M_{4}}\right)+O\left(\log ^{+} T(r, S)\right)
$$

for $r$ outside a set $E$ of finite linear measure. 
Now, we use (16) and (19) to obtain

$$
\begin{aligned}
T(r, S) & =N(r, S)+m(r, S) \\
& \leq N(r)+m(r, S) \\
& \leq O\left(r^{M_{5}}\right)+O\left(\log ^{+} T(r, S)\right)
\end{aligned}
$$

and so

$$
T(r, S)=O\left(r^{M_{6}}\right)
$$

for $r$ outside a set $E$ of finite linear measure. This proves Claim 2 .

Claim 3: We claim that

$$
T(r, S) \leq O\left(r^{M_{6}}\right), \quad \text { for all large } r
$$

This follows from Claim 1, [5, Lemma 1.1.1] and the fact that $T(r, S)$ is nondecreasing.

Now, dividing (15) by $L$ shows that if at $z$ the function $\frac{w^{\prime}}{w}$ has a pole, then either

$$
A_{2}=\sum_{m=0}^{k}\left[\left(\begin{array}{l}
k \\
m
\end{array}\right) \frac{L^{(m)}}{L} R_{k-m}+\sum_{j=1}^{k-2}\left(\begin{array}{l}
j \\
m
\end{array}\right) B_{j} \frac{L^{(m)}}{L} R_{j-m}\right]=0
$$

or

$$
A_{1}=\sum_{m=0}^{k}\left[\left(\begin{array}{l}
k \\
m
\end{array}\right) \frac{L^{(m)}}{L} Q_{k-m}+\sum_{j=1}^{k-2}\left(\begin{array}{l}
j \\
m
\end{array}\right) B_{j} \frac{L^{(m)}}{L} Q_{j-m}\right]+A=\infty
$$

Using Claim 3, we can write (15) as

$$
A_{1}+A_{2} \frac{w^{\prime}}{w}=0
$$

Where $T\left(r, A_{j}\right)=O\left(r^{M_{7}}\right), j=1,2$ and $A_{2} \neq 0$ by the assumption of Case (III).

Now, by using Claim 1 and (20), we get

$$
\begin{aligned}
N\left(r, \frac{1}{w}\right) & =N\left(r, \frac{w^{\prime}}{w}\right) \\
& \leq N\left(r, \frac{1}{A_{2}}\right)+N\left(r, A_{1}\right) \\
& =O\left(r^{M_{8}}\right) .
\end{aligned}
$$

So, $N\left(r, \frac{1}{w}\right)$ has finite order. But this contradicts (4). Hence, Case (III) cannot occur.

\section{Proof of Theorem 1.3}

Assume the hypotheses of Theorem 1.3. Taking $k=2$ in Theorem 1.2, two cases have to be considered as in [1].

In case (a): $L$ is a constant and $A=P$ by (7) and Lemma 1.1. 
In case (b): $L$ is not constant, but (8) and Lemma 1.1 give

$$
0=\sum_{m=0}^{2}\left(\begin{array}{l}
2 \\
m
\end{array}\right) L^{(m)} R_{2-m}=L R_{2}+2 L^{\prime} R_{1}+L^{\prime \prime} R_{0}=2 L^{\prime} .
$$

But this requires that $L$ should be constant, a contradiction.

\section{Proof of Theorem 1.4}

Assume the hypotheses of Theorem 1.4. Taking $k=3$ and $B_{1}=B$ in Theorem 1.2, we have two cases to consider as in [1].

In case (a), $L$ is a constant, and (7) and Lemma 1.1 give $A=P^{\prime}$. But, since $w$ solves (1) and (11), we have

$$
0=w^{\prime \prime \prime}+B w^{\prime}+A w=w^{\prime \prime \prime}+B w^{\prime}+P^{\prime} w=w^{\prime \prime \prime}+P^{\prime} w+P w^{\prime},
$$

which gives $P=B$.

In case (b), $L$ is not constant and, using (8) and Lemma 1.1,

$$
0=\sum_{m=0}^{3}\left[\left(\begin{array}{l}
3 \\
m
\end{array}\right) L^{(m)} R_{3-m}+\left(\begin{array}{l}
1 \\
m
\end{array}\right) B_{1} L^{(m)} R_{1-m}\right]=-P L+B L+3 L^{\prime \prime}
$$

and therefore

$$
L^{\prime \prime}=\frac{1}{3} P L-\frac{1}{3} B L .
$$

Differentiating (21), we obtain

$$
L^{\prime \prime \prime}=\frac{1}{3} P^{\prime} L+\frac{1}{3} P L^{\prime}-\frac{1}{3} B^{\prime} L-\frac{1}{3} B L^{\prime} .
$$

Also, we have, using (22),

$$
\begin{aligned}
A & =-\sum_{m=0}^{3}\left[\left(\begin{array}{c}
3 \\
m
\end{array}\right) \frac{L^{(m)}}{L} Q_{3-m}+\left(\begin{array}{c}
1 \\
m
\end{array}\right) B_{1} \frac{L^{(m)}}{L} Q_{1-m}\right] \\
& =P^{\prime}+3 P \frac{L^{\prime}}{L}-B \frac{L^{\prime}}{L}-\frac{L^{\prime \prime \prime}}{L} \\
& =P^{\prime}+3 P \frac{L^{\prime}}{L}-B \frac{L^{\prime}}{L}-\frac{1}{3} P^{\prime}-\frac{1}{3} P \frac{L^{\prime}}{L}+\frac{1}{3} B^{\prime}+\frac{1}{3} B \frac{L^{\prime}}{L} \\
& =\frac{8}{3} P \frac{L^{\prime}}{L}+\frac{2}{3} P^{\prime}+\frac{1}{3} B^{\prime}-\frac{2}{3} B \frac{L^{\prime}}{L} .
\end{aligned}
$$

\section{Proof of Theorem 1.5}

We will need the following lemma, which is due to Bank and Laine $[6,8]$.

Lemma 5.1 Let B an entire function. Then, every solution of the equation

$$
u^{\prime \prime \prime}+4 B u^{\prime}+2 B^{\prime} u=0
$$

is of the form $u=y_{1} y_{2}$, where $y_{1}, y_{2}$ are solutions (possibly linearly dependent) of

$$
\gamma^{\prime \prime}+B y=0 .
$$


- If $y_{1}, y_{2}$ are linearly dependent, then $u=y^{2}$ with $y$ a solution of (24) and

$$
B=\frac{-y^{\prime \prime}}{y}=\frac{1}{4}\left(\frac{u^{\prime}}{u}\right)^{2}-\frac{1}{2} \frac{u^{\prime \prime}}{u} .
$$

- If $y_{1}, y_{2}$ are linearly independent, then

$$
4 B=\left(\frac{u^{\prime}}{u}\right)^{2}-2 \frac{u^{\prime \prime}}{u}-\frac{k^{2}}{u^{2}}
$$

where $k=W\left(y_{1}, y_{2}\right)$.

We remark that (25) is the well known Bank-Laine product formula [6].

Now, assume the hypotheses of Theorem 1.5. Taking $k=4$ and $B_{1}=B_{2}=0$ in Theorem 1.2, there are two cases have to be considered.

Case (a): $L$ is a constant and, by using (3), we have

$$
A=-Q_{4}=-P^{2}+P^{\prime \prime}
$$

But, differentiating (1) two times gives

$$
0=w^{(4)}+\left(P^{\prime \prime}-P^{2}\right) w+2 P^{\prime} w^{\prime}
$$

Since we also have $w^{(4)}+A w=0$, this gives

$$
0=2 P^{\prime} w^{\prime}
$$

So $P$ must be constant, but this contradicts our assumption that $P$ is transcendental. Hence, Case (a) cannot occur.

Case (b): $L$ is non-constant and $L$ satisfies, using (3),

$$
\begin{aligned}
0 & =\sum_{m=0}^{4}\left(\begin{array}{c}
4 \\
m
\end{array}\right) L^{(m)} R_{4-m} \\
& =4 L^{\prime \prime \prime}-4 L^{\prime} P-2 L P^{\prime}
\end{aligned}
$$

and therefore

$$
L^{\prime \prime \prime}=L^{\prime} P+\frac{1}{2} L P^{\prime}
$$

Since this is a linear differential equation and $P$ is an entire function, it follows that $L$ is an entire function.

By using (3) and (9), we obtain

$$
\begin{aligned}
A & =-\sum_{m=0}^{4}\left(\begin{array}{c}
4 \\
m
\end{array}\right) \frac{L^{(m)}}{L} Q_{4-m} \\
& =-P^{2}+P^{\prime \prime}+4 P^{\prime} \frac{L^{\prime}}{L}+6 P \frac{L^{\prime \prime}}{L}-\frac{L^{(4)}}{L} .
\end{aligned}
$$


Differentiating (26) and dividing by $L$ gives

$$
\frac{L^{(4)}}{L}=P \frac{L^{\prime \prime}}{L}+\frac{3}{2} P^{\prime} \frac{L^{\prime}}{L}+\frac{1}{2} P^{\prime \prime} .
$$

By substituting this in (27), we get

$$
\begin{aligned}
A & =-P^{2}+P^{\prime \prime}+4 P^{\prime} \frac{L^{\prime}}{L}+6 P \frac{L^{\prime \prime}}{L}-P \frac{L^{\prime \prime}}{L}-\frac{3}{2} P^{\prime} \frac{L^{\prime}}{L}-\frac{1}{2} P^{\prime \prime} \\
& =5 P \frac{L^{\prime \prime}}{L}+\frac{5}{2} P^{\prime} \frac{L^{\prime}}{L}+\frac{1}{2} P^{\prime \prime}-P^{2} .
\end{aligned}
$$

Therefore, (26) and (28) prove (13).

Suppose that $w, v$ have the same zeros. Then, $L$ has no zeros and poles.

Now, we set $B=\frac{-P}{4}$ in (26) and apply Lemma 5.1. Then, $L=y_{1} y_{2}$ where $y_{1}, y_{2}$ are solutions of (24).

Then, we have the following two cases:

Case 1: If $y_{1}, y_{2}$ are linearly dependent, then $L=y^{2}$ with $y$ a solution of (24) and

$$
\frac{-P}{4}=\frac{1}{4}\left(\frac{L^{\prime}}{L}\right)^{2}-\frac{1}{2} \frac{L^{\prime \prime}}{L}, \quad P=-\left(\frac{L^{\prime}}{L}\right)^{2}+2 \frac{L^{\prime \prime}}{L} .
$$

Let $L=e^{2 C}$ with $C$ an entire function. Then,

$$
P=-\left(2 C^{\prime}\right)^{2}+2\left(2 C^{\prime \prime}+4 C^{\prime 2}\right)=4\left(C^{\prime 2}+C^{\prime \prime}\right) .
$$

Substituting these in (28) shows that $A$ is a differential polynomial in $C$.

Case 2: If $y_{1}, y_{2}$ are linearly independent, then

$$
P=-\left(\frac{L^{\prime}}{L}\right)^{2}+2 \frac{L^{\prime \prime}}{L}+\frac{k^{2}}{L^{2}}
$$

where $k=W\left(y_{1}, y_{2}\right)$. Also, $L$ is not a polynomial, since $P(\infty) \neq 0$.

Let $L=e^{C}$ with $C$ an entire function. Then,

$$
\begin{aligned}
P & =-C^{\prime 2}+2 C^{\prime \prime}+2 C^{2}+k^{2} e^{-2 C} \\
& =2 C^{\prime \prime}+C^{\prime 2}+k^{2} e^{-2 C} .
\end{aligned}
$$

Substituting these in (28) shows that $A$ is a differential polynomial in $e^{-C}$ and $C$.

\section{Acknowledgements}

The author would like to thank his supervisor Prof. Jim Langley for his support and guidance. Also, he would like to thank King Abdulaziz University for financial support for his PhD study.

\section{Author details}

'Department of Mathematics, Faculty of Education, King Abdulaziz University, P.O. Box 15758, Jeddah 21454, Saudi Arabia ${ }^{2}$ School of Mathematical Sciences, University of Nottingham, Nottingham NG72RD, UK

\section{Competing interests}

The author declares that they have no competing interests.

Received: 24 July 2011 Accepted: 7 December 2011 Published: 7 December 2011

\section{References}

1. Asiri, A: Common zeros of the solutions of two differential equations. Comput Methods Funct Theory. 12(1), 67-85 (2012) 
2. Wittich, H: Neuere Untersuchungen über eindeutige analytische Funktionen. Ergebnisse der Mathematik und ihrer Grenzgebiete. Springer, Berlin, Göttingen, Heidelberg8 (1955)

3. Hayman, WK: Meromorphic functions. Oxford Mathematical Monographs. Clarendon Press, Oxford (1964)

4. Hille, E: Ordinary differential equations in the complex domain. Dover Publications Inc., Mineola, New York (1997) [Reprint of the 1976 original]

5. Laine, I: Nevanlinna theory and complex differential equations. de Gruyter Studies in Mathematics. Walter de Gruyter \& Co, Berlin15 (1993)

6. Bank, SB, Laine, I: On the oscillation theory of $\mathbb{\otimes}+A f=0$ where $A$ is entire. Trans Am Math Soc. 273, 351-363 (1982)

7. Alotaibi, A: On complex oscillation theory. Results Math. 47, 165-175 (2005)

8. Bank, SB, Laine, I: Representations of solutions of periodic second order linear differential equations. J Reine Angew Math. 344, 1-21 (1983)

9. Bank, SB, Laine, I, Langley, JK: On the frequency of zeros of solutions of second order linear differential equations. Results Math. 10, 8-24 (1986)

10. Bank, SB, Laine, I, Langley, JK: Oscillation results for solutions of linear differential equations in the complex plane. Results Math. 16, 3-15 (1989)

11. Bank, SB, Langley, JK: Oscillation theory for higher order linear differential equations with entire coefficients. Complex Var Theory Appl. 16(2-3), 163-175 (1991)

12. Langley, JK: Some oscillation theorems for higher order linear differential equations with entire coefficients of small growth. Results Math. 20(1-2), 517-529 (1991)

13. Langley, JK: On entire solutions of linear differential equations with one dominant coefficient. Analysis 15(2), 187-204 (1995). [Corrections in: Analysis 15(4):433 (1995)]

14. Rossi, J: Second order differential equations with transcendental coefficients. Proc Am Math Soc. 97, 61-66 (1986). doi:10.1090/S0002-9939-1986-0831388-8

doi:10.1186/1029-242X-2011-134

Cite this article as: Asiri: Common zeros of the solutions of two differential equations with transcendental coefficients. Journal of Inequalities and Applications 2011 2011:134.

\section{Submit your manuscript to a SpringerOpen ${ }^{\odot}$} journal and benefit from:

Convenient online submission

- Rigorous peer review

- Immediate publication on acceptance

- Open access: articles freely available online

- High visibility within the field

- Retaining the copyright to your article

Submit your next manuscript at $\gg$ springeropen.com 